\title{
Buildup of steady-state picosecond pulses in an actively mode-locked laser-diode array
}

\author{
Jahn-Chung Kuo, C.-S. Chang, and Ci-Ling Pan
}

Institute of Electro-optical Engineering, National Chiao Tung University, Hsinchu, Taiwan 300, China

Received April 22, 1991

\begin{abstract}
We have studied experimentally the temporal and spectral characteristics of an actively mode-locked laserdiode array in an external cavity as it evolves to the steady state. It is found that the buildup time to steady. state picosecond pulses takes less than 45 round trips. The number of clusters of the longitudinal-mode spectrum reduces during the evolution and finally approaches a steady-state spectral distribution with the pulse energy mainly distributed among a few clusters near the line center.
\end{abstract}

Much effort has been directed to the study of actively mode-locked semiconductor lasers. ${ }^{1-7}$ Active mode locking of the laser-diode array has also been reported..$^{8-10}$ Most of these studies, however, concentrated on the steady-state characteristics, and relatively less attention has been paid to the transient buildup mechanisms. Using a fast photodiode and the sampling oscilloscope, AuYeung et al. ${ }^{11}$ observed that an actively mode-locked doubleheterostructure GaAlAs laser took approximately 30 round trips to reach the steady state. Their results, however, were limited by the bandwidth of the detection system. In a numerical study, Demokan ${ }^{12}$ found that the pulse evolution time of an actively mode-locked GaAlAs/GaAs laser with one perfectly antireflection-coated facet would be $\simeq 150$ round trips. Recently, Blixt and Krotkus ${ }^{13}$ showed both theoretically and experimentally that an actively mode-locked InGaAsP buried-heterostructure laser diode reached its steady state in 40 to 50 round trips. In their study the laser diode was biased with rectangular pulses of different durations. The pulse duration measured corresponded to an average of the mode-locked pulses from the beginning to the end of the rectangular pulse. As a result, the pulse evolution during the initial stage of the buildup was not known. In this Letter we report what are to our knowledge the first results on the evolution to the steady state of the pulse widths and the corresponding spectra of an actively mode-locked laserdiode array in an external cavity by time-gating techniques.

A block diagram of our experimental setup is shown in Fig. 1. We used a commercial 10-stripe phase-locked laser-diode array (Spectral Diode Laboratories SDL-2419C, $\lambda=0.81 \mu \mathrm{m}$ ) as the gain medium. It had a high-reflectivity coating of $95 \%$ on the back facet and an antireflection coating of $\simeq 0.1 \%$ reflectivity on the front facet. A $20 \times, 0.5$ N.A. microscope objective lens and a cylindrical lens $(f=150 \mathrm{~mm})$ imaged the antireflection-coated facet of the laser-diode chip onto an output mirror $(R=40 \%)$. The laser was dc biased at $250 \mathrm{~mA}$ $\left(I_{\mathrm{th}}=260 \mathrm{~mA}\right)$ and injected simultaneously with a rf sinusoidal modulation signal of as much as $1 \mathrm{~W}$ at $433 \mathrm{MHz}$. To minimize the optical pulse width, the frequency of the $\mathrm{rf}$ modulation signal was slightly detuned below the frequency matched to the external-cavity round-trip time. A train of stable single pulses with a FWHM of $45 \mathrm{ps,} \mathrm{assuming}$ Gaussian pulse shapes, could be routinely obtained. The average output power was $15 \mathrm{~mW}$. In order to investigate the transient behavior of the actively mode-locked laser-diode array, the $\mathrm{rf}$ sinusoidal signal was chopped by a fast microwave P-I-N diode switch (Hewlett-Packard 33144A). The output of the P-I-N switch was a $433-\mathrm{MHz}$ signal that had a square envelope with a repetition rate of $0.5 \mathrm{MHz}$, a duty cycle of $50 \%$, and a rise time of $30 \mathrm{~ns}$. The laser output was fed to a noncollinear autocorrelator or a monochromator and detected by a gatable photomultiplier tube (PMT) with a gate width of $10 \mathrm{ns.} \mathrm{A}$ boxcar integrator with a 50-ns gate was used for signal averaging. By delaying these two synchronous gates with respect to the onset of diode-laser action, we can sample the temporal and spectral characteristics of the laser as it evolves to the steady state.

Figure 2 illustrates the pulse widths of the actively mode-locked external-cavity laser-diode array as a function of delay time. Zero delay time corresponded to the onset of diode-laser action. A typical autocorrelation trace iof a steady-state mode-locked pulse is shown in the inset of Fig. 2. The buildup can be qualitatively described as follows: Since the dc bias was below the threshold, initially there was only spontaneous emission inside the cavity. As the P-I-N diode switch was turned on, a sinusoidal signal superimposed onto the dc bias was also injected into the laser-diode array. A train of weakly modulated optical pulses would appear. As these weak pulses circulated inside the cavity, the central regions of the pulses would experience more gain than their wings. As a result, the pulse shapes sharpened as the pulse energies built up. During the first $40 \mathrm{~ns}$, the pulse width shortened quickly and approached the steady state in approximately $100 \mathrm{~ns}$. This corresponded to 45 round trips. The laser output power reached the steady state at the same time. 


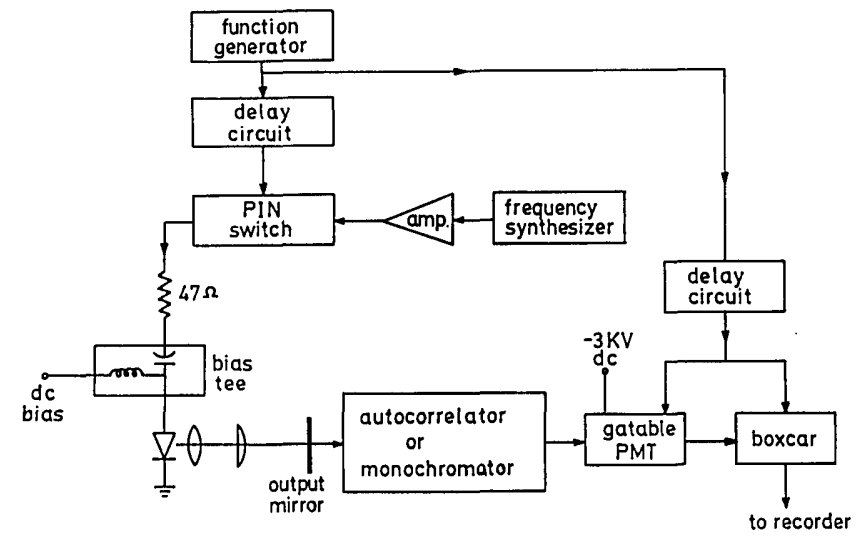

Fig. 1. Block diagram of the experimental arrangement. PMT, photomultiplier tube; amp., amplifier.

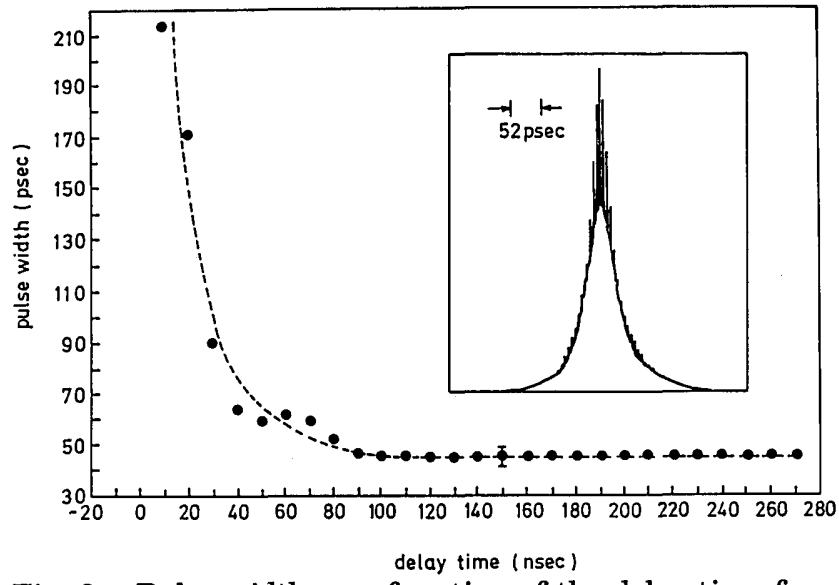

Fig. 2. Pulse width as a function of the delay time from the onset of laser-diode array action. The inset shows an autocorrelation trace of the steady-state laser pulse.

This is consistent with the observation of Blixt and Krotkus. ${ }^{13}$ An overshoot behavior at the beginning of the buildup was also observed. Since our P-I-N diode switch had a rise time of $30 \mathrm{~ns}$, the actual buildup time to the steady state of our test laser should be shorter than 45 round trips. This result was somewhat shorter than those reported in previous studies (Refs. 12 and 13) for solitary laser diodes at 0.83 and $1.3 \mu \mathrm{m}$, respectively. Buildup of optical pulses in mode-locked lasers with other types of gain medium takes even more round trips. For a passive colliding-pulse mode-locked ring Rhodamine 110/HICI dye laser, Williams et al. ${ }^{14}$ reported that the pulse widths and the corresponding spectra evolved to the steady state in approximately $12.5 \mu \mathrm{s}$, which corresponds to 1300 round trips. We have also measured a buildup time of $\simeq 15 \mu \mathrm{s}$, i.e., approximately 1500 round trips, for a Rhodamine 6G/DODCI colliding-pulse mode-locked laser system. ${ }^{15}$ Using a mode-locked argon-ion laser to synchronously pump the Rhodamine $6 \mathrm{G}$ dye laser, Kasinski et al. ${ }^{16}$ found that the buildup time to steady-state picosecond pulses took approximately $10 \mu$ s, i.e., 800 round trips. A recent study on an additive-pulse mode-locked $\mathrm{Ti}: \mathrm{Al}_{2} \mathrm{O}_{3}$ laser showed that ultrashort mode-locked pulses evolved from mode-beating intensity fluctuations in approximately $200 \mu \mathrm{s}$, or 16,000 round trips. ${ }^{17}$
The FWHM of the corresponding spectra as a function of the delay time is shown in Fig. 3. Intuitively one would expect the spectra to broaden as the laser pulse shortens and approaches the steady state. It was observed instead that the bandwidth of the spectrum also narrowed quickly during the initial stage of the buildup $(\simeq 40 \mathrm{~ns})$ and then reached a steady state in approximately 100 ns. Figure 4 shows a series of spectra that correspond to the points labeled (a)-(d) in Fig. 3. The spacing of the clusters of longitudinal modes of the chip cavity was $\simeq 0.3 \mathrm{~nm}$, corresponding to a chip length of $250 \mu \mathrm{m}$ with a group velocity of $c / 4.3$. It should be noted that the average spectra of the laser output consist of multiple clusters, and the number of clusters was reduced as the pulse widths narrowed. This can be explained by the following: Numerical studies by Garside and $\mathrm{Kempf}^{6}$ have shown that multiple reflections between the two facets of the chip would still be present even if the residual reflectivity of

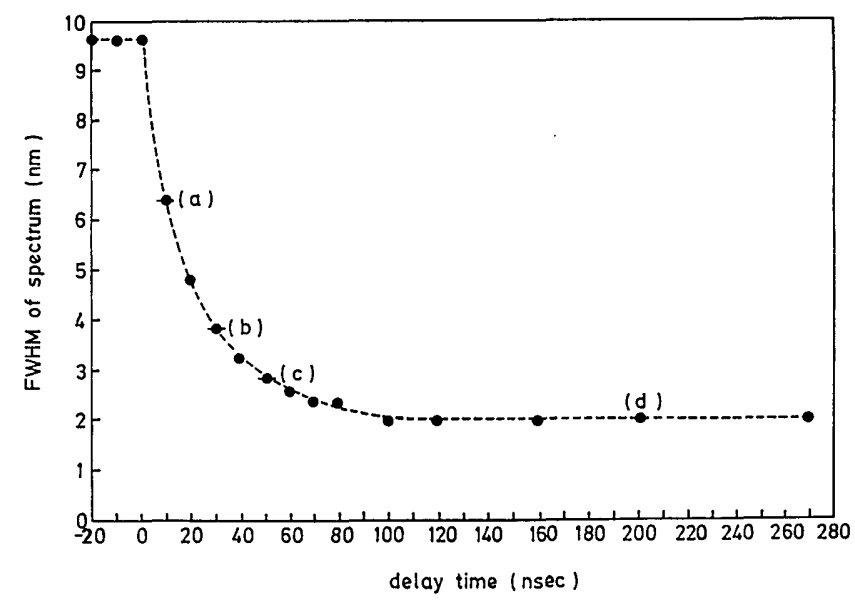

Fig. 3. The FWHM of the laser spectrum as a function of the delay time.

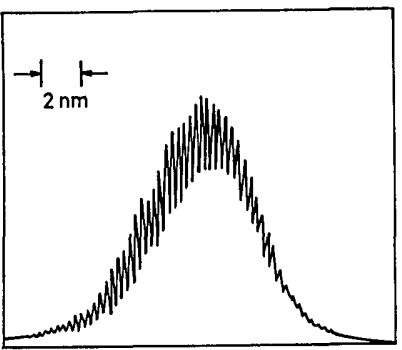

(a)

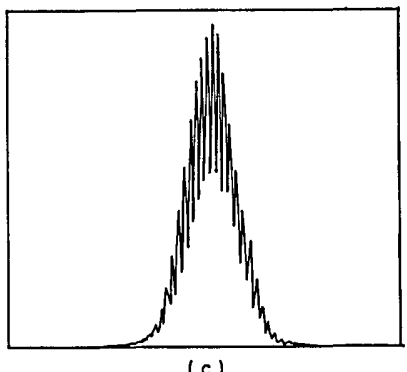

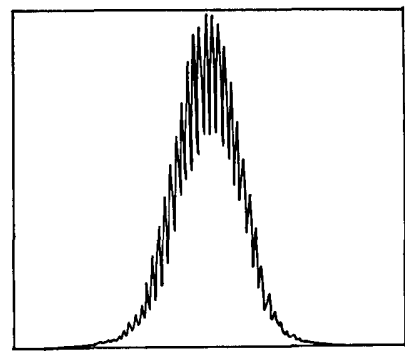

(b)

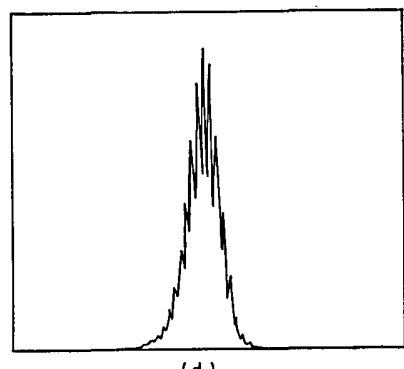

(d)
Fig. 4. Actual spectra of the laser at points labeled (a)-(d) in Fig. 3. 
the diode facet facing the external-cavity mirror were low. This effect gave rise to a laser output spectrum that exhibited multiple clusters. During the initial stage of the pulse formation, the unlocked spectrum translated into random fast fluctuations in time and longer pulse durations, and several diode chip cavity modes would build up. After a few round trips, the pulse shapes monotonously shortened and cleaned up. At the same time, the number of clusters in the spectrum would also decrease owing to the effect of competition among the clusters in a homogeneous broadened medium. Finally, the pulses approached the steady state with the energy of the entire pulse distributed among a few clusters of the longitudinal modes of the chip cavity near the line center. During the pulse evolution, the pulse durations gradually narrowed, and we expect the bandwidth of each of the clusters to also broaden. Owing to the resolution limitation of our monochromator, however, we did not measure the variations of cluster bandwidth as a function of the delay time at this time.

In summary, we have presented detailed data on the temporal and spectral evolution of picosecond pulses generated by an actively mode-locked laserdiode array in an external cavity. The buildup to the steady state takes approximately 45 round trips, if we neglect the rise time of the P-I-N diode switch. The number of clusters of the longitudinal-mode spectrum reduces during the evolution and finally approaches a steady-state spectral distribution with the pulse energy mainly distributed among a few clusters near the line center. This is a manifestation of homogeneous broadening. Initially, the longitudinal modes are unlocked, and random and fast fluctuations in time correspond to a broad output spectrum. As the pulses clean up, the spectrum narrows. At the same time, the pulse width also approaches its steady-state value.
This research was partially supported by the National Science Council of the Republic of China under grants NSC80-0417-E009-05 and NSC800417-E009-17. The authors thank the reviewer for valuable comments.

\section{References}

1. S. W. Corzine, J. E. Bowers, G. Przybylek, U. Koren, B. I. Miller, and C. E. Soccolich, Appl. Phys. Lett. 52, 348 (1988).

2. P. T. Ho, L. A. Glasser, E. P. Ippen, and H. A. Haus, Appl. Phys. Lett. 33, 241 (1978).

3. J. P. van der Ziel, J. Appl. Phys. 52, 4435 (1981).

4. J. C. AuYeung, IEEE J. Quantum Electron. QE-17, 398 (1981).

5. J. C. Goodwin and B. K. Garside, IEEE J. Quantum Electron. QE-19, 1068 (1983).

6. B. K. Garside and P. Kempf, Appl. Opt. 25, 3846 (1986).

7. J. E. Bowers, P. A. Morton, A. Mar, and S. W. Corzine, IEEE J. Quantum Electron. 25, 1426 (1989).

8. J. P. van der Ziel, H. Temkin, R. D. Dupuis, and R. M. Mikulyak, Appl. Phys. Lett. 44, 357 (1984).

9. L. G. Joneckis and P.-T. Ho, in Digest of Conference on Lasers and Electro-Optics (Optical Society of America, Washington, D.C., 1988), paper WM4.

10. H. Masuda and A. Takada, Electron. Lett. 25, 1418 (1989).

11. J. C. AuYeung, L. A. Bergman, and A. R. Johnson, Appl. Phys. Lett. 41, 124 (1982).

12. M. S. Demokan, Int. J. Electron. 60, 67 (1986).

13. P. Blixt and A. Krotkus, Opt. Quantum Electron. 22, 561 (1990).

14. J. A. R. Williams, P. M. W. French, and J. R. Taylor, IEEE J. Quantum Electron. 26, 1434 (1990).

15. J. C. Kuo and C. L. Pan, Opt. Lett. 15, 1297 (1990).

16. J. J. Kasinski, L. A. Gomez-Jahn, R. J. Dwayne Miller, P. Geist, B. Geoffroy, F. Heisel, A. Martz, and J. A. Miehe, J. Opt. Soc. Am. B 3, 1566 (1986).

17. J. Goodberlet, J. Wang, J. G. Fujimoto, and P. A. Schulz, Opt. Lett. 15, 1300 (1990). 